\title{
Original
}

\section{Clinical Relevance of Quantitaive Measurements of Drug-induced Extrapyramidal Symptoms}

\author{
Yoichi Aкimoto, Masaru Mimura, \\ Shintaro Nukada and Kunitoshi KamiJima
}

\begin{abstract}
We devised and tested a simple and practical measuring system for assessing drug-induced extrapyramidal signs (EPS) in schizophrenic patients. We first assessed the ability of the system to clinically measure the degree of EPS, and then investigated differences between conventional and atypical antipsychotic agents. Schizophrenic patients (28 males, 22 females) were compared with age-matched normal controls. A 14-item battery of measurements was analyzed in terms of established EPS scale scores and psychiatric symptoms. Measurements concentrated on the face, upper and lower limbs. Patients were impaired in 10 out of 14 quantitative subtests concerning movements when compared with normal controls. Facial characteristics that differed between patients and normal controls included oral agility and Myerson's sign; for the upper limbs included various fine, dominant-handed tasks; and for the lower limbs included gait. We then compared 16 patients receiving conventional agents and 18 patients receiving atypical agents, using the same system. Improvements in oral agility and fine finger movements were observed for patients on newer atypical agents. Our measurement system can reveal motor dysfunction and difficulties that cause problems in daily life for schizophrenic patients, and therefore provides a clinically useful assessment of drug-induced EPS. The most sensitive subtests for detecting drug-induced side effects were those that dealt with oral agility and rapid, repetitive finger movements.
\end{abstract}

Key words : extrapyramidal symptoms, schizophrenia, antipsychotic drugs, adverse effects, movement disorders

\section{Introduction}

While advances in antipsychotic agents have markedly improved the prognosis of patients with schizophrenia, they are still plagued by well-known adverse side effects. These include movement disorders, sedation, seizures, cardiovascular and liver dysfunction, anticholinergic symptoms, weight gain, sexual dysfunction, and agranulocytosis ${ }^{1}$. Extrapyramidal symptoms (EPS) are the most common side effects of antipsychotic agents. Drug-induced EPS include akathisia, muscle spasms (dystonia), involuntary grimacing or chewing movements (dyskinesia), and parkinsonism, which involves symptoms of tremor, rigidity, and bradykinesia. These movement disorders may occur in isolation or as part of a mixed Department of Neuropsychiatry, Showa University School of Medicine, 1-5-8 Hatanodai, Shinagawa-ku, Tokyo 1428666, Japan. 
syndrome.

Up to $90 \%$ of patients treated with conventional antipsychotic drugs such as haloperidol, chlorpromazine, or levomepromazine may develop EPS, while approximately $20 \%$ of patients develop tardive dyskinesia ${ }^{2)}$. These drug-induced movement disorders are a serious burden for patients and their caregivers and EPS may lead to social rejection, difficulty with rehabilitation, and poor employment prospects. In addition, EPS may compromise treatment efficacy by inducing noncompliance ${ }^{3)}$. As EPS can also limit dosages, the development of newer antipsychotic agents has focused on reducing the risk of EPS as well achieving effective symptom control. Although newer "atypical" antipsychotics, such as risperidone, olanzapine, and quetiapine, cause far fewer EPS than conventional antipsychotics, EPS still represent a major problem for patients being treated for schizophrenia.

Several measures have been devised to quantitatively evaluate the severity of drug-induced EPS. These include the Extrapyramidal Symptom Rating Scale (ESRS), the St. Hans Rating Scale ${ }^{4)}$, and the Drug Induced Extra-Pyramidal Symptoms Scale (DIEPSS) ${ }^{5)}$, and they cover a wide range of the EPS spectrum. More specifically, the Barnes Akathisia Rating Scale ${ }^{6}$, the Hillside Akathisia Scale ${ }^{7)}$, and the Rating Scale for Acute Drug-Induced Akathisia $^{8)}$, were designed to evaluate akathisia. The Simpson-Angus scale (SAS $)^{9)}$ focuses on parkinsonism and the Abnormal Involuntary Movement Scale (AIMS) ${ }^{10)}$ has been used widely for tardive dyskinesia. Although the aforementioned scales are useful for clinical evaluation, we set out to devise a simple, more objective measure for quantitative assessment. Analyses using a rating scale pose difficulties such as inter-rater reliability and even inter-session reliability with the same rates. Training of raters could be a crucial factor. Some studies have proposed more quantitative analyses of movement disorders. For example, Gattaz and Buchel ${ }^{11)}$ used digital image processing to convert analog movements representing tardive dyskinesia into a digital signal; they found this method useful for early detection of drug side effects. Giovannoni et $a l^{12)}$ proposed a bradykinesia-akinesia incoordination (BRAIN) test, as a simple, rapid computer-assisted keyboard test able to quantify upper limb motor function. In this study we examined the ability of our newly devised battery of motor measurements to clinically assess the degree of drug-induced EPS.

\section{Methods}

\section{Study 1: Clinical applicability of our system}

\section{Subjects}

Fifty schizophrenic patients ( 28 males, 22 females) were compared with gender- and age-matched normal controls. All subjects were right-handed. Table 1 shows demographic and clinical background data for the subjects. Positive and negative psychotic symptoms were evaluated with the Brief Psychiatric Rating Scale (BPRS) ${ }^{13)}$ and the Positive and Negative Symptoms Scale (PANSS) ${ }^{14)}$. Overall scores on both scales and the scores for positive symptoms on the PANSS were higher in females. Basic EPS were rated using the to the DIEPSS ${ }^{5)}$. All patients received either conventional or atypical antipsychotic agents and $90.1 \%$ of patients $(46 / 51)$ also received anticholinergic antiparkinsonian agents. The total dose of antipsychotic medication was converted to haloperidol equivalents, while the total dose of antiparkinsonian drugs was converted to biperiden equivalents. There was no difference between female and male patients in terms of duration of illness, dose of antipsychotic agents, or dose of antiparkinsonian agents. 
Table 1. Demographic data of each subject group in study 1 and clinical data for schizophrenic patients

\begin{tabular}{llcccc}
\hline Group & \multicolumn{2}{c}{ Schizophrenia } & & Control \\
Gender & M & F & M & F \\
\hline Number & & 28 & 22 & 20 & 22 \\
Age (years) & $45.1(11.3)$ & $49.6(13.9)$ & $46.5(16.3)$ & $47.1(12.7)$ \\
\hline Disease duration (months) & $24.7(14.0)$ & $27.1(15.1)$ & - & - \\
BPRS & & $31.4(6.6)$ & $45.6(11.3)^{* *}$ & - & - \\
PANSS & Positive & $11.9(3.6)$ & $17.5(5.7)^{* *}$ & - & - \\
\multicolumn{1}{c}{ Negative } & $20.9(4.5)$ & $23.0(6.0)$ & - & - \\
DIEPSS & General & $34.9(5.1)$ & $43.6(11.1)^{* *}$ & - & - \\
Medication & HPD eq. (mg) & $6.4(3.4)$ & $6.1(2.3)$ & - & - \\
& Biperiden eq. (mg) & $3.6(3.5)$ & $4.8(2.7)$ & - & - \\
\hline
\end{tabular}

${ }^{* *} P<0.01$, significantly higher than for male patients.

Table 2. Measures of EPS

\begin{tabular}{|c|c|c|}
\hline \multirow[t]{5}{*}{ Face } & $1-1$ & Number of spontaneous eye blinks $/ 30 \mathrm{sec}$ \\
\hline & $1-2$ & Number of blinks in response to Myerson's test (glabellar tapping) \\
\hline & $2-1$ & Number of involuntary tongue movements $/ 30 \mathrm{sec}$ \\
\hline & $2-2$ & Number of involuntary mouth movements $/ 30 \mathrm{sec}$ \\
\hline & 3 & Oral agility: number of "pa-ta-ka" repetition $/ 30 \mathrm{sec}$ \\
\hline \multirow[t]{4}{*}{ Upper limbs } & 4 & Thumb movements: number of presses of a counter button $/ 30 \mathrm{sec}$ \\
\hline & 5 & Number of small beans picked up and moved by chopsticks \\
\hline & $6-1$ & Speed of drawing triangles (number $/ 30 \mathrm{sec}$ ) \\
\hline & $6-2$ & Size change of triangles drawn in $6-1$ \\
\hline \multirow[t]{5}{*}{ Lower limbs } & 7 & Time required to stand up $(\mathrm{sec})$ \\
\hline & 8 & Time required to initiate walking $(\mathrm{sec})$ \\
\hline & $9-1$ & Steps required to walk $5 \mathrm{~m}$ \\
\hline & $9-2$ & Time required to walk $5 \mathrm{~m}(\mathrm{sec})$ \\
\hline & 10 & Time required to turn to the right $(\mathrm{sec})$ \\
\hline
\end{tabular}

\section{Procedure}

We devised a quantitative scale measuring movements of the face, upper limbs, and lower limbs: Subtests for the face included eye-blink frequencies, number of involuntary mouth and tongue movements, oral agility, and Myerson's sign. Subtests for the upper limbs included frequencies of rapid thumb movements (pressing a counter button), picking up and moving small beans using chopsticks, and drawing triangles of similar size as quickly as possible. Subtests for the lower limbs included time required to stand up, initiate walking and make turns. Speed and steps required for walking $5 \mathrm{~m}$ were also determined. Details of subtests are shown in Table 2. 


\section{Statistical analyses}

Each measure was subjected to analysis of variance (ANOVA) to compare motor function between patients and controls. ANOVAs were conducted in a $2 \times 2$ manner (schizophrenia vs. control and female vs. male) because previous reports had suggested gender differences in EPS ${ }^{15)}$. Female and male subjects were also analyzed separately using a post-hoc Bonferroni comparison. Scores of movement measures were also examined for correlation with psychotic symptom variables (BPRS and PANSS) and basic EPS ratings (DIEPSS).

\section{Study 2: Conventional vs. atypical antipsychotics}

\section{Subjects}

Thirty-four right-handed schizophrenic patients were included in the study ( 18 females, 16 males). All patients were taking antipsychotic agents (16 conventional, 18 atypical). Anticholinergic antiparkinsonian agents were used in $81.3 \%$ of patients (13/16) receiving conventional drugs, and in $44.4 \%$ of patients (8/18) receiving atypical drugs. Total doses of antipsychotic drugs and antiparkinsonian drugs were expressed in haloperidol and biperiden equivalents, respectively. Table 3 shows demographic and clinical background data for the subjects. No significant differences were obtained between conventional and atypical groups or between female and male groups regarding duration of illness, or dose of antipsychotic medication. However, doses of antiparkinsonian agents were significantly higher for patients treated with conventional agents, compared to those receiving atypicals $[F(1,30)=4.18, P<0.05]$. Neither the main effect of gender, or drug $\times$ gender interaction, reached significance. Separate analyses for female and male patients did not yield any differences in the doses of antiparkinsonian agents between conventional and atypical groups. Procedure

Patients underwent the same quantitative assessment of movements of the face, upper limbs, and lower limbs as in study 1.

\section{Statistical analyses}

Each measure was submitted to individual ANOVA; also, 2 (conventional vs. atypical drug) $\times 2$ (female vs. male) ANOVAs were conducted. Female and male subjects were also analyzed separately using post-hoc Bonferroni comparison.

\section{Results}

Study 1: Clinical applicability of our system

Assessment of motor symptoms

Table 3. Demographic and clinical data for schizophrenic patients in study 2

\begin{tabular}{lccccc}
\hline Drug & \multicolumn{2}{c}{ Conventional } & \multicolumn{2}{c}{ Atypical } \\
Gender & M & F & M & F \\
\hline Number & 8 & 8 & 8 & 10 \\
Age (years) & $33.4(8.7)$ & $31.4(7.3)$ & $29.2(7.1)$ & $31.1(4.7)$ \\
Disease duration (months) & $36.5(9.0)$ & $28.5(14.6)$ & $17.1(14.1)$ & $24.4(15.2)$ \\
Antipsychotics HPD eq. (mg) & $7.2(7.8)$ & $11.9(14.9)$ & $8.7(4.0)$ & $11.0(7.7)$ \\
Antiparkinson Biperiden eq. (mg) & $2.7(3.4)$ & $2.9(2.1)$ & $1.1(0.4)$ & $1.8(0.6)$ \\
\hline
\end{tabular}


Quantitative measurements of movement are shown in Table 4. Differences between patients and controls reached statistical significance in 10 of the 14 subtests. For facial movements, significant differences between groups were obtained in 2 of 5 subtests [Myerson, $\mathrm{F}(1,88)=9.12, \mathrm{P}<0.01$; and oral agility, $\mathrm{F}(1,88)=42.9, P<0.0001]$. For upper limb movements, all 4 subtests showed patient/control differences: pressing a counter, $F$ $(1,88)=25.03, P<0.0001$; picking up beans with chopsticks, $F(1,88)=100.0, P<0.0001$; speed of drawing triangles, $F(1,88)=19.69, \quad P<0.0001 ;$ and size of triangles, $F$ $(1,88)=5.45, P<0.05$. Similarly, for lower limb movements, patient/control differences were obtained in all 5 subtests: standing up, $F(1,88)=4.53, P<0.05$; initiation of walking, $\mathrm{F}(1,88)=15.50, P=0.0002$; number of steps, $\mathrm{F}(1,88)=30.67, P<0.0001$; walking speed, $\mathrm{F}$ $(1,88)=12.10, P<0.001$; and turning speed, $\mathrm{F}(1,88)=20.24, P<0.0001$.

Some subtests demonstrated gender differences. For facial movements, the main effects of gender were obtained for the Myerson sign $[F(1,88)=6.34, P<0.02]$ and for oral agility $[F(1,88)=4.99, P<0.03]$. For upper limb movements, the main effects of gender were significant for pressing a counter $[F(1,88)=17.70, P<0.0001]$ and for picking up beans with chopsticks $[F(1,88)=3.41, P<0.05]$. For lower limb movements, gender differences were significant in initiation of walking $[F(1,88)=7.04, P=0.01]$, and making turns $[F$ $(1,88)=5.94, P<0.05]$. For most subtests, group $\times$ gender interactions were not significant, indicating that male subjects scored better than female subjects irrespective of group status. The only exception was for Myerson's sign; male schizophrenic patients showed substantially more blinks in response to tapping than male controls [group $\times$ gender interaction, $F$ $(1,88)=11.53, P=0.001]$. Separate patient/control group comparisons using post-hoc Bonferroni analyses are shown in Table 4 for female and male subjects.

Correlations between our system, BPRS, PANSS, and DIEPSS

Quantitative movement measurements showed few correlations with psychotic symptoms (Table 5). Specifically, negative symptoms had no significant correlation with quantitative measurements of movement, except for a negative correlation with pressing a counter. Some of the movement subtests showed mild correlations with DIEPSS subscores. Bradykinesia showed correlations with oral agility $(-0.35, P<0.01)$, counter pressing $(-0.37, P<0.01)$, and turning $(-0.43, P<0.01)$. Sialorrhea correlated with involuntary tongue movement $(0.31, P<0.05)$ and bean manipulation $(-0.31, P<0.05$. $)$. Muscle rigidity correlated with the Myerson sign $(0.49, P<0.001)$ and with turning $(-0.35, P<0.05)$, akathisia with bean manipulation $(0.43, P<0.01)$, dystonia with bean manipulation $(0.34, P<0.05)$, and dyskinesia with oral agility $(0.35, P<0.05)$. However, overall severity and the total scores from the DIEPSS did not show significant correlations with quantitative measurements of movement.

\section{Study 2: Conventional vs. atypical antipsychotics}

Data from the quantitative measurements of movement are shown in Table 6 . Correlation with the class of drug used was significant in 3 of the 14 subtests. For facial movements, a significant difference between conventional and atypical drugs was obtained in the oral agility subtest $[F(1,30)=5.78, P=0.02]$. For upper limb movements there was a significant difference between drugs, when using the 'counter pressing' subtest [F $(1,30)=4.61, P<0.05]$. The ability to draw triangles of uniform size tended to differ between conventional and atypical groups $[F(1,30)=3.05, P<.10]$. For lower limb 
Table 4. Results of quantitative movement examination

\begin{tabular}{|c|c|c|c|c|c|c|c|c|}
\hline \multirow[b]{2}{*}{ Gender } & \multicolumn{4}{|c|}{ Schizophrenia } & \multicolumn{4}{|c|}{ Control } \\
\hline & \multicolumn{2}{|r|}{$\mathbf{M}$} & \multicolumn{2}{|r|}{$\mathbf{F}$} & \multicolumn{2}{|c|}{$\mathbf{M}$} & \multicolumn{2}{|c|}{$\mathbf{F}$} \\
\hline \multicolumn{9}{|l|}{ Face } \\
\hline Blink（spontaneous） & 8.6 & $(10.3)$ & 8.8 & $(8.0)$ & 5 & $(6.7)$ & 5.3 & $(3.7)$ \\
\hline Myerson & 5.3 & $(5.8)^{* * *}$ & 1.4 & $(1.0)$ & 1.0 & $(1.0)$ & 1.7 & $(1.2)$ \\
\hline Tongue (involuntary) & 0.6 & $(1.7)$ & 0.0 & $(0.0)$ & 0.0 & $(0.0)$ & 0.0 & $(0.0)$ \\
\hline Mouth (involuntary) & 0.7 & $(2.0)$ & 0.3 & $(0.7)$ & 0.0 & $(0.0)$ & 0.0 & $(0.0)$ \\
\hline Oral agility & 40.8 & $(13.3)^{* * *}$ & 37.6 & $(11.1)^{* * *}$ & 58.9 & $(8.2)$ & 51.3 & $(12.1)$ \\
\hline \multicolumn{9}{|l|}{ Upper limb } \\
\hline Counter & 99.8 & $(29.7)^{* * *}$ & 83.0 & $(22.3)^{* * *}$ & 136.9 & $(37.8)$ & 104.4 & $(18.3)$ \\
\hline Beans & 14.0 & $(4.1)^{* * *}$ & 12.9 & $(4.9)^{* * *}$ & 24.5 & $(5.3)$ & 22.0 & $(4.6)$ \\
\hline Triangle speed & 10.9 & $(5.3)^{* * *}$ & 12.0 & $(6.7)^{*}$ & 17.7 & $(6.7)$ & 16.5 & $(5.9)$ \\
\hline Size & 19.8 & $(8.0)$ & 21.6 & $(7.8)^{*}$ & 22.4 & $(9.3)$ & 27.3 & $(8.8)$ \\
\hline \multicolumn{9}{|l|}{ Lower limb } \\
\hline Stand up ( $\sec )$ & 3.6 & $(2.9)$ & 3.9 & $(2.1)$ & 2.4 & $(0.4)$ & 3.4 & $(1.1)$ \\
\hline Walk initiation $(\mathrm{sec})$ & 0.98 & $(0.64)^{*}$ & 0.79 & $(0.29)^{* * *}$ & 0.67 & $(0.20)$ & 0.38 & $(0.36)$ \\
\hline Walking : steps $/ 5 \mathrm{~m}$ & 8.4 & $(1.9)^{* *}$ & 9.2 & $(1.1)^{* * *}$ & 7.2 & $(1.1)$ & 7.3 & $(1.1)$ \\
\hline Walking speed, $5 \mathrm{~m}(\mathrm{sec})$ & 6.4 & $(5.7)$ & 6.6 & $(2.1)^{* * *}$ & 4.1 & $(0.7)$ & 3.9 & $(0.7)$ \\
\hline Turning speed $(\mathrm{sec})$ & 1.7 & $(0.9)^{* *}$ & 2.2 & $(0.9)^{* * *}$ & 1.1 & $(0.6)$ & 1.4 & $(0.5)$ \\
\hline
\end{tabular}

${ }^{*} P<0.05,{ }^{* *} P<0.01$, or ${ }^{* * *}<0.001$ compared to corresponding control subjects.

Table 5. Correlation of quantitative movement tests with schizophrenia and EPS scales

\begin{tabular}{|c|c|c|c|c|c|}
\hline & \multirow[t]{2}{*}{ BPRS } & \multicolumn{3}{|l|}{ PANSS } & \multirow[t]{2}{*}{ DIEPSS } \\
\hline & & Positive & Negative & General & \\
\hline \multicolumn{6}{|l|}{ Face } \\
\hline Blink（spontaneous） & - & - & - & - & - \\
\hline Myerson & $-0.40^{*}$ & $-0.45^{* *}$ & - & - & - \\
\hline Tongue (involuntary) & - & - & - & - & - \\
\hline Mouth (involuntary) & - & - & - & - & - \\
\hline Oral agility & - & - & - & - & - \\
\hline \multicolumn{6}{|l|}{ Upper limbs } \\
\hline Counter & - & - & $-0.46^{* *}$ & - & - \\
\hline Beans & - & - & - & - & - \\
\hline Triangle speed & $-0.42 * *$ & $-0.40^{*}$ & - & - & - \\
\hline Triangle size & - & - & - & - & - \\
\hline \multicolumn{6}{|l|}{ Lower limbs } \\
\hline Stand up & - & - & - & - & - \\
\hline Walk initiation & $-0.37^{*}$ & $-0.35^{*}$ & - & $-0.53^{* *}$ & - \\
\hline Steps $/ 5 \mathrm{~m}$ & - & - & - & - & - \\
\hline Walking speed & - & - & - & - & - \\
\hline Turning speed & - & - & - & - & - \\
\hline
\end{tabular}

${ }^{*} P<0.05,{ }^{* *} P<0.01$ 
Table 6. Results of quantitative movement examination for study 2

\begin{tabular}{|c|c|c|c|c|c|}
\hline \multirow{2}{*}{\multicolumn{2}{|c|}{$\begin{array}{l}\text { Drug type } \\
\text { Gender }\end{array}$}} & \multicolumn{2}{|c|}{ Conventional } & \multicolumn{2}{|c|}{ Atypical } \\
\hline & & $\mathbf{M}$ & $\mathrm{F}$ & $\mathbf{M}$ & $\mathbf{F}$ \\
\hline Number & & 8 & 8 & 8 & 10 \\
\hline \multicolumn{6}{|l|}{ Face } \\
\hline \multicolumn{2}{|c|}{ Blink（spontaneous） } & $9.4(7.7)$ & $9.1(5.6)$ & $10.1(11.0)$ & $11.7(6.5)$ \\
\hline \multicolumn{2}{|l|}{ Myerson } & $2.1(2.0)$ & $2.4(2.5)$ & $2.2(1.7)$ & $1.2(0.6)$ \\
\hline \multicolumn{2}{|c|}{ Tongue (involuntary) } & $0.0(0.0)$ & $0.6(1.8)$ & $0.0(0.0)$ & $0.0(0.0)$ \\
\hline \multicolumn{2}{|c|}{ Mouth (involuntary) } & $0.6(1.8)$ & $0.6(1.8)$ & $0.0(0.0)$ & $0.0(0.0)$ \\
\hline \multicolumn{2}{|c|}{ Oral agility } & $42.7(7.2)^{*}$ & $37.0(11.8)$ & $49.9(7.9)$ & $45.4(9.9)$ \\
\hline \multicolumn{6}{|l|}{ Upper limb } \\
\hline \multicolumn{2}{|l|}{ Counter } & $130.9(11.3)$ & $120.8(23.0)^{*}$ & $136.1(25.6)$ & $144.8(16.8)$ \\
\hline \multicolumn{2}{|l|}{ Beans } & $14.1(3.6)$ & $15.6(4.6)$ & $14.9(4.0)$ & $16.6(4.2)$ \\
\hline \multirow[t]{2}{*}{ Triangle } & Speed & $11.8(4.2)$ & $11.4(5.3)$ & $9.8(6.7)$ & $12.9(7.1)$ \\
\hline & Size & $25.1(10.3)$ & $23.9(6.0)^{* *}$ & $26.0(7.8)$ & $30.2(3.7)$ \\
\hline \multicolumn{6}{|l|}{ Lower limb } \\
\hline \multicolumn{2}{|c|}{ Standing up (sec) } & $2.6(0.8)$ & $2.7(1.1)$ & $2.5(0.6)$ & $2.9(0.5)$ \\
\hline \multicolumn{2}{|c|}{ Walk initiation $(\mathrm{sec})$} & $0.3(0.1)$ & $0.6(0.3)$ & $0.3(0.2)$ & $0.4(0.3)$ \\
\hline \multicolumn{2}{|c|}{ Walking; steps $5 \mathrm{~m}$} & $8.0(0.6)$ & $9.1(1.9)$ & $8.3(1.7)$ & $8.1(1.0)$ \\
\hline \multicolumn{2}{|c|}{ Walking speed $5 \mathrm{~m}(\mathrm{sec})$} & $4.9(0.6)$ & $6.0(1.9)$ & $4.7(1.3)$ & $5.2(1.8)$ \\
\hline \multicolumn{2}{|c|}{ Turning（sec） } & $1.8(0.5)$ & $2.1(0.6)$ & $1.9(0.5)$ & $1.8(0.4)$ \\
\hline
\end{tabular}

${ }^{*} P<0.05$ or ${ }^{* *} P<0.01$, with conventional drugs associated with poorer performance than atypical drugs.

movements, no differences between drug types were significant in any subtest. A main effect of gender was significant only for the limb subtest that measured initiation time for walking $[F(1,30)=6.44, P<0.02]$. Drug $x$ gender interaction did not reach statistical significance in any of the subtests.

\section{Discussion}

Study 1 demonstrated that hospitalized chronic schizophrenic patients who received treatment with antipsychotic agents were impaired in 10 of the14 subtests in our quantitative system for evaluating movements. Three subtests that were ineffective for discriminating patient from control groups concerned spontaneous involuntary eyelid, tongue, and oral movements. Therefore, although oral dyskinesia and facial spasms are the most frequent involuntary movements among chronic schizophrenia patients, observation may not be an adequate quantitative measure for discriminating pathologic movements. Some intervention by the examiner was required for the 10 subtests that successfully discriminated patients from normal controls. Further, 9 of these 10 subtests (all except for Myerson's sign) involved voluntary movements, such as speaking, drawing, grasping, and walking.

Only weak correlations were observed between movement measurements and psychotic symptoms. One could argue that significant negative symptoms may impair voluntary movements of schizophrenic patients. However, negative symptoms did not account for the overall impairment as shown by the quantitative assessment of movements. Three subtests (Myerson's sign, speed of drawing triangles, and speed of initiation of walking) showed 
negative correlations with positive symptoms as indexed by the BPRS and by the PANSS positive-symptom scores. An impulsive tendency may partially account for negative correlations, but the relationship between positive symptoms and EPS is not clear in the present study. Moreover, our sample showed differences in positive symptoms between female and male patients. Therefore, a study of a larger patient group is needed, as gender differences for positive symptoms are less likely to be significant.

One unexpected finding in study 1 was that quantitative measures of movement showed only weak correlations with the DIEPSS, a widely used rating scale for overall EPS severity. Recent studies concerning brain injury and neurocognitive rehabilitation have emphasized discrepancies between functional impairment and disability. Patients with noticeable impairments on functional tests may not experience any significant disability in active daily life, and conversely, functional tests may fail to uncover severe disability in a real-life setting. Considering this dissociation between functional impairment scores and disability, our measurements of movements may be more closely related to real-life disabilities. Future studies are needed to explore this possibility. We conclude that our testing system can detect motor dysfunction and problems with activities of daily living for schizophrenic patients. As such, it represents a practical method for clinically assessing drug-induced EPS.

Study 2 demonstrated that atypical antipsychotics agents are superior to conventional antipsychotics in terms of minimizing treatment-related EPS, consistent with previous studies documenting the advantage of atypical agents ${ }^{16,17)}$. When relatively young schizophrenic patients received approximately 26.5 months of treatment with antipsychotics, differences were seen between conventional and atypical agents. In particular, atypicals had less side effects involving oral and upper limb voluntary movements (oral agility and fine finger movements such as pressing a counter and drawing). These rapid repetitive movements were most sensitive for detecting drug-induced side effects.

The atypical agent most frequently used in study 2 was risperidone $[77.8 \%(14 / 18)$ of the patients in the atypical agent group]. In a meta-analysis of 11 double-blind risperidone trials, Fleishhacker et $a^{18)}$ reported that side effect profiles based on qualitative analysis by ESRS showed no differences between placebo and risperidone groups receiving 1 to $2 \mathrm{mg}$ /day. Parkinsonism, tremor, akathisia, and sialorrhea were more likely to occur with haloperidol than with placebo or risperidone at 1 to $6 \mathrm{mg} /$ day. A risperidone dose of 4 $\mathrm{mg} /$ day (equivalent to $8 \mathrm{mg} /$ day of haloperidol) is widely considered to be optimal for most patients with schizophrenia. Lower doses may be appropriate for patients being treated for a first psychotic episode ${ }^{19)}$. In this sense, the doses of atypical agents in study 2 may be higher than necessary. This may partially explain the relatively small differences in EPS between conventional and atypical agents in this study.

Our tests are brief, simple, and easy to perform as part of a clinical examination and we believe that they add valuable information to knowledge obtained using rating scales such as ESRS and DIEPSS. The limitations of our study include a relatively small number of subjects and a large number of drugs used. One question that remains unanswered concerns the possible differences between individual drugs, particularly specific atypical agents. For example, some studies report similar EPS profiles for risperidone and olanzapine ${ }^{20)}$ while others report significantly fewer EPS in olanzapine-treated patients compared with risperidone-treated patients ${ }^{21}$. In our present study, only $16.6 \%$ of atypical-agent patients (3/18) were treated with olanzapine, which precluded meaningful comparisons between 
risperidone and olanzapine. However, our quantitative assessment of movements may have more power than previous rating scales in characterizing individual atypical agents. Future studies are needed with larger numbers of subjects taking the drugs that are to be compared.

One important and intriguing question concerns the impact of EPS on the quality of life of schizophrenic patients in real-life settings. As suggested by study 1, our quantitative testing system may be particularly sensitive to disabilities that interfere with everyday communication, toileting, feeding and recreation. Since we did not perform analyses in terms of established instruments for evaluating activities of daily living or quality of life, this crucially important issue awaits future studies.

\section{Acknowledgements}

The authors are deeply indebted to Drs. Kentaro Matsumaru, Taro Takahashi, Asako Kosuga, and Hiroki Yamada for their help in recruiting and examining patients.

\section{References}

1) Collaborative Working Group on Clinical Trial Evaluations : Adverse effects of the atypical antipsychotics. $J$ Clin Psychiatry 59(suppl 12): 17-22 (1998)

2) Casey DE : Effects of clozapine therapy in schizophrenic individuals at risk for tardive dyskinesia. $J$ Clin Psychiatry 59(suppl 3) : 31-37 (1998)

3) Gerlach $\mathbf{J}$ : The continuing problem of extrapyramidal symptoms: strategies for avoidance and effective treatment. J Clin Psychiatry 60(suppl 23) : 20-24 (1999)

4) Gerlach J, Korsgaard S, Clemmesen P, Lauersen AM, Magelund G, Noring U, Povlsen UJ, Bech P and Casey DE : The St. Hans Rating Scale for extrapyramidal syndromes: reliability and validity. Acta Psychiatr Scand $87: 244-252$ (1993)

5) Inada T and Yagi G : Current topics in neuroleptic-induced extrapramidal symptoms in Japan. Keio J Med 45 : 95-99 (1996)

6) Barnes TRE : A rating scale for drug-induced akathisia. Br J Psychiatry 154 : 672-676 (1989)

7) Fleischhacker WW, Miller CH, Schett P, Barnas C and Ehrmann H: The Hillside Akathisia Scale : a reliability comparison of the English and German Versions. Psychopharmacology 105 : 141-144 (1991)

8) Sachdev P: A rating scale for acute drug-induced akathisia : development, reliability, and validity. Biol Psychiatry 35 : 263-271 (1994)

9) Beasley CM Jr, Tollefson GD, and Tran PV: Safety of olanzapine. J Clin Psychiatry 58(suppl 10) : 13-17 (1997)

10) National Institute of Mental Health : Abnormal Involuntary Movement Scale (AIMS). In : ECDEU Assessment manual for psychopharmacology (Revised) Guy W (Ed) U.S. Department of Health, Education, and Welfare, Public Health Service, Rockville, pp. 534-537 (1976)

11) Gattaz WF and Buchel C: Assessment of tardive dyskinesia by means of digital image processing. Psychopharmacology 111 : 278-284 (1993)

12) Giovannoni G, van Schalkwyk J, Fritz VU and Lees AL: Bradykinesia akinesia incoordination test (BRAIN TEST): an objective computerised assessment of upper limb motor function. $J$ Neurol Neurosurg Psychiatry 67 : 624-629 (1999)

13) Kolakowska T : Brief psychiatric rating scale. Glossary and rating instructions. Oxford University Press, Oxford (1976)

14) Kay SR, Opler LA and Fiszbein A: Positive and Negative Syndrome Scale (PANSS) Rating Manual. Multi-Health System Inc, Toronto (1991)

15) Yassa R and Jeste DV : Gender differences in tardive dyskinesia: A critical review of the literature. Schizophr Bull 18 : 701-715 (1992)

16) Leucht S, Pitschel-Walz G, Abraham D, and Kissling W : Efficacy and extrapyramidal side-effects of the new antipsychotics olanzapine, quetiapine, risperidone, and sertindole compared to conventional antipsychotics and placebo. A meta-analysis of randomized controlled trials. Schizophr Res 35 : 51-68 (1999)

17) Tarsy D, Baldessarini RJ, and Tarazi FI : Effects of newer antipsychotics on extrapyramidal function. CNS Drugs 16 : 23-45 (2002)

18) Fleischhacker WW, Lemmens $P$ and van Baelen B : A qualitative assessment of the neurological safety of antipsychotic drugs; an analysis of a risperidone database. Pharmacopsychiatry 34 : 104-110 (2001)

19) Lemmens P, Brecher $M$ and Van Baelen B: A combined analysis of double-blind studies with risperidone vs. 
placebo and other antipsychotic agents: factors associated with extrapyramidal symptoms. Acta Psychiatr Scand 99 : 160-170 (1999)

20) Conley RR and Mahmoud R : A randomized double-blind study of risperidone and olanzapine in the treatment of schizophrenia or schizoaffective disorder. Am J Psychiatry 158 : 765-774 (2001)

21) Tran PV, Hamilton SH, Kuntz AJ, Potvin JH, Andersen SW, Beasley C Jr and Tollefson GD : Double-blind comparison of olanzapine versus risperidone in the treatment of schizophrenia and other psychotic disorders. J Clin Psychopharmacol 17 : 407-418 (1997)

[Received December 26, 2002 : Accepted January 17, 2003] 